\title{
Escape the Matrix
}

\author{
Ein mathematischer Escape Room
}

\author{
Birgit Komander und Dirk Lorenz
}

\begin{abstract}
Sie haben mit Ihrer Lerngruppe bis spät in die Nacht für Ihre Klausur in Linearer Algebra in einem Seminarraum in der Uni gelernt. Wie schon öfter, sind Sie über das viele Lernen eingeschlafen und wachen nun morgens im Seminarraum auf. Der Pförtner hat über Nacht die Tür abgeschlossen und in einer halben Stunde beginnt die Klausur! Schaffen Sie es noch rechtzeitig, die Tür wieder zu öffnen um die Klausur anzutreten?
\end{abstract}

Drei Studierende sind eingeschlossen in einem Seminarraum in der TU Braunschweig. Im Raum befindet sich ein Whiteboard, entlang einer Wand steht wie immer eine Reihe Sideboards und in einer Ecke stehen ein paar Mathe-Bücher. Außerdem sind zwei Folien an der Wand. Auf den zweiten Blick enthält der Raum noch ein paar mehr Dinge, die normalerweise nicht da sind: Eine Werkzeugkiste steht in der Ecke, unter einem Tisch steht ein Rollcontainer, hinter einem Vorsprung steht eine Holzkiste, woanders im Raum eine kleine Schatztruhe. Der Rollcontainer, der Werkzeugkasten und die Holzkisten sind allesamt mit Schlössern verschlossen und auf den Folien an der Wand steht etwas geschrieben. Der Monitor an der Wand zeigt einen Countdown der herunterzählt. Die Studierenden betrachten die Folien und sehen auf den ersten Blick ratlos aus. Doch plötzlich scheinen sie etwas zu verstehen und beginnen Zahlenkombinationen an der Schlössern auszuprobieren. Nach und nach kommen Rätsel zum Vorschein, Bücher werden durchgeblättert, weitere Schlösser werden geöffnet ...

Die Studierenden befinden sich in einem mathematischen Escape Room. Sie haben sich freiwillig einschließen lassen und stellen sich der Herausforderung, anhand von Rätseln aus der Linearen Algebra den Raum zu entschlüsseln und schließlich den Schlüssel für die Tür zu finden und damit das Spiel zu gewinnen.

Was ist ein Escape Room?

Escape Rooms gibt es mittlerweile in vielen Städten Deutschlands. Man kann solch ein Spiel auch in Form eines kleinen Brettspiels in sein Wohnzimmer holen. Das Prinzip ist stets das Gleiche: Eine kleine Menschengruppe versucht innerhalb einer vorgegebenen Zeit alle Rätsel zu lösen, um das Spiel zu gewinnen. Bei den Brettspielen kann es zum
Beispiel um das Finden eines Gegenstands innerhalb des Spiels gehen oder das Auflösen eines Verbrechens.

In einem „live“ Escape Room lässt sich eine kleine Menschengruppe freiwillig in einem Raum einschließen, um innerhalb der Zeit mithilfe von im Raum versteckten Hinweisen und Rätseln wieder zu entkommen. Die verschiedenen Escape Rooms sind meist thematisch um eine Hintergrundgeschichte gestrickt. Mal muss man aus einen Gefängnis ausbrechen, mal einen Raketenstart verhindern oder in das Büro eines Bankdirektors einbrechen (vgl. [2]). Die gestellten Rätsel können verschiedener Natur sein, wie Geschicklichkeitsspiele, um an einen Gegenstand zu gelangen oder auch Knobelaufgaben, um beispielsweise einen Zahlencode für ein Schloss zu erhalten. Manchmal reicht es aus sich nur genau im Raum umsehen, um versteckte Gegenstände wie einen Schlüssel zu finden.

\section{Warum einen mathematischen Escape Room?}

In Escape Rooms stellen sich die Teilnehmer einer Herausforderung. Sie müssen unter Zeitdruck, der durch eine Spielgeschichte, Kulisse oder auch Hintergrundmusik noch verschärft wird, knifflige Aufgaben lösen und haben daran auch noch Spaß. Eine Herausforderung ist das Mathematikstudium allemal. Knifflige Aufgaben haben wir in der Mathematikausbildung zu genüge, und nicht selten kommt bei den Studierenden auch Zeitdruck dazu. Der Spaß kommt allerdings manchmal zu kurz. Mit dem mathematischen Escape Room wollten wir also das Studium um die Komponente Spaß ergänzen (wir hatten schon in früheren Semestern gemerkt, dass „Recreational Mathematics“ Studierende motivieren kann [3]). Wir haben zwar keine Beispiele für mathematische Escape Rooms an Universitäten gefunden (sehr wohl aber deren Einsatz in Schulen auf
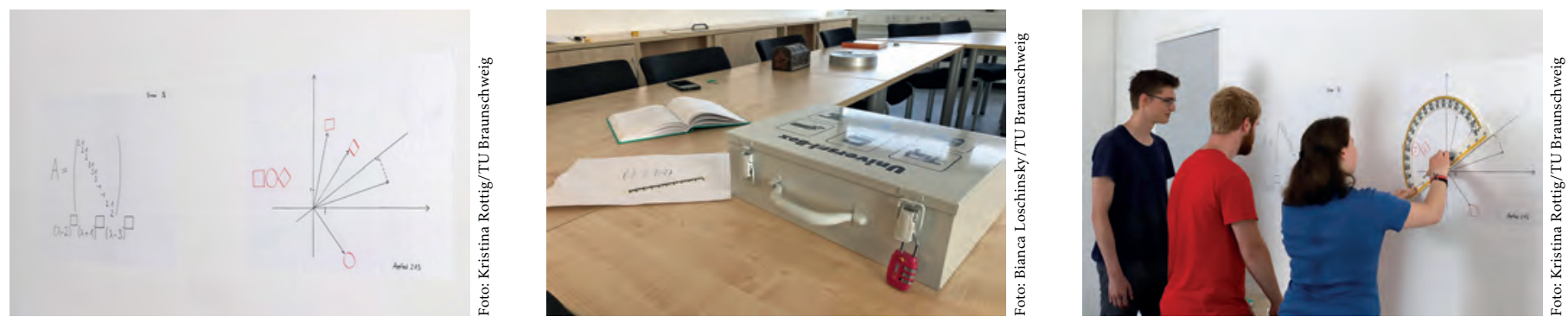
verschiedenen Niveaus), aber die Idee scheint in anderen Fächern schon eingesetzt zu werden (siehe z. B. [4] für ein Beispiel aus der Physik). Tatsächlich gibt es auch wissenschaftliche Literatur zu Escape Rooms und deren Einsatz in der Lehre [1].

Wie macht man einen mathematischen Escape Room?

Die Grundelemente für einen Escape Room sind

- ein geeigneter Raum, den man möglichst frei einrichten kann und der möglichst nichts mehr enthält, was nicht zum Spiel gehört,

- eine Spielgeschichte, die erklärt, warum die Spielerinnen und Spieler im Raum sind, und was sie da erreichen sollen,

- Rätsel, die gelöst werden müssen,

- eine Möglichkeit, das Vorgehen im Raum zu beobachten und auch um Rückmeldung und Hilfestellung von außen an die Spielerinnen und Spieler zu geben.

Bei uns bot sich der Seminarraum des Instituts an. Dieser ist spärlich eingerichtet, hatte neben Tischen, Stühlen, einem Whiteboard und vier abschließbaren Schränken auch noch einen an der Wand installierten Monitor mit einem Rechner, der auch mit dem Netz verbunden ist. Da wir den Escape Room für den Anfang nur für die Studierenden aus der Vorlesung „Lineare Algebra“ als Ergänzung zur Prüfungsvorbereitung anbieten wollten, war unsere Spielgeschichte schnell klar (sie steht direkt unter der Überschrift dieses Artikels).

Nun mussten wir uns Aufgaben und Rätsel überlegen, die thematisch aus der Linearen Algebra stammen. Diese mussten wir weiter so verpacken, dass die Lösungen der Aufgaben weiter verwertet werden konnten, also beispielsweise einen Code liefern, die ein Zahlenschloss öffnen kann. Außerdem mussten wir uns über die technischen Details klar werden. Wie genau sollte die Hilfestellung an die Spieler funktionieren? Und wie genau sollten wir überhaupt mitbekommen, dass Hilfe angebracht ist?

Die Rätsel waren schnell erstellt. Hier eine Matrix in Jordanscher Normalform, für die das Minimalpolynom abgelesen werden sollte; die Exponenten der faktorisierten Form gaben den Code. Da ein lineares Gleichungssystem, deren Lösung einen Ortsvektor angibt. Zusammen mit einem Koordinatensystem zeigte dieser auf einen Code unter vielen, die wir in einen verschlossenen Schrank mit Kreppband geklebt haben. Weiter erstellten wir ein Projektionsrätsel. In einem $\mathbb{R}^{2}$ Koordinatensystem zeichneten wir einen linearen Unterraum und Vektoren, die Länge der orthogo- nalen Projektionen der drei Vektoren ergab den Zahlencode. Ein weiteres Rätsel bestand aus einer Uhr und drei verschiedenen komplexen Zahlen. Die Uhr sollte dabei den Einheitskreis darstellen. Die komplexen Zahlen sollten mit den Zahlen der Uhr in Verbindung gebracht werden, um wiederum einen weiteren Zahlencode zu erhalten. Wir merkten schnell, dass die Lineare Algebra eine Vielzahl an möglichen Rätseln bot, um Zahlenkombinationen in die praktische Umsetzung zu integrieren.

Danach machten wir uns an die technische Umsetzung der Hilfestellungen. Die Grundidee war, den schon vorhandenen Bildschirm im Raum zu nutzen, um Hinweise in verschriftlichter Form neben einem runter laufenden Countdown anzuzeigen; ein kurzes Pythonskript ermöglichte genau dies. Wir brachten eine Webcam in einer Ecke des Raums an, von der man den Großteil der Rätsel gut im Blick hatte. Den Computer im Besprechungsraum verbanden wir über SSH mit einem Notebook im Nebenraum, der unsere Schaltzentrale werden sollte.

Die Technik schien zu funktionieren, über eine SkypeVerbindung konnten wir den Raum gut mithilfe der Webcam sehen und im Notfall auch über das Mikrofon einen Hinweis geben. Wir bereiteten den Raum mit allen Rätseln vor: Auf Whiteboardfolien, die elektrostatisch an der Wand haften, waren die beiden Anfangsrätsel abgebildet. Alle weiteren Rätsel verschlossen wir in den vorhandenen Schränken. Bücher verteilten wir im Raum. Einige Hinweise waren bei den Rätseln, die auf bestimmte Seiten in Büchern hinwiesen, wie z. B. das Minimalpolynom. Beim Aufbau erstellten wir parallel ein Flowchart, damit wir zwischen den Durchgängen den Raum schnell wieder auf den Anfangszustand bringen konnten.

Der Testlauf mit freiwillig gemeldeten Mitarbeitern der Mathematik konnte starten. Der Anfang lief gut, die Spieler kamen gut mit den ersten Rätseln zurecht. Doch dann brach die Skype-Verbindung ab und wir wussten nicht mehr, was im Raum vor sich ging. Nach den 30 Minuten öffneten wir die Tür, die Gruppe war weit gekommen, aber hatte eine Sache übersehen. Wir holten uns Rückmeldung ein und konnten dank der konstruktiven Kritik noch einige Rätsel verbessern. Auch an der Technik schraubten wir noch, sodass diese einwandfrei lief.

\section{Wie ist es gelaufen?}

An dem Tag, an dem „Escape the Matrix“ stattfinden sollte, waren neun verschiedene Teams angemeldet. Alle Teams hatten sich kreative Teamnamen überlegt, wie „Matrixin-
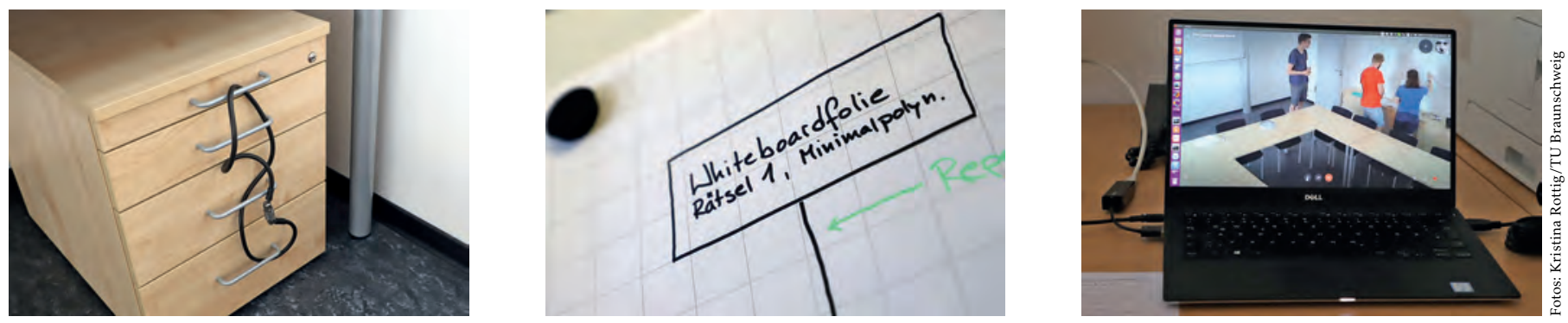
vasion“, „Die Freunde des chinesischen Restsatzes“ oder „Die Algebros ft. Herr C.“. Wir stellten einen Warteraum zur Verfügung in dem wir die Regeln für den Escape Room und einen High-Score mit der Restzeit, die der Countdown nach erfolgreichem Verlassen des Raums anzeigen würde, an die Tafel schrieben.

Den Escape Room selbst hatten wir bereits am Abend vorher vollständig präpariert. Im Nebenbüro bereiteten wir die Schaltzentrale mit Notebooks, Mikrofon und unserem Flowchart vor. Ein letzter Systemcheck und das Spektakel konnte beginnen.

Das erste Team „Matrixinvasion“, ein Team unserer Zweitsemester-Studierenden der Linearen Algebra-Vorlesung, wartete bereits vor dem Warteraum. Sie schienen ähnlich gespannt wie wir zu sein, auf das, was auf sie zukommen würde.

Wir führten sie durch den Flur zum Besprechungsraum, der noch geschlossen war und erklärten ihnen nochmal die Regeln. (Zum Beispiel, dass es nicht notwendig ist, Möbel $\mathrm{zu}$ verrücken und dass alle Schlösser im Raum nur durch Schlüssel oder Codes geöffnet werden durften). Nachdem wir ihnen die Spielgeschichte erzählt hatten, brachten wir sie in den Raum, schlossen sie ein und starteten den Countdown. ${ }^{1}$ Im Nebenbüro setzten wir uns gespannt vor den Bildschirm und fieberten mit der „Matrixinvasion“ mit.

Nach kurzen Anlaufschwierigkeiten lösten sie dann die ersten beiden Rätsel im Raum, doch die halbe Stunde Zeit reichte leider nicht aus, um erfolgreich dem Escape Room zu entkommen.

Wir erklärten den Studierenden noch schnell, welche Rätsel es noch gab und wie sie zu lösen gewesen wären, schließlich sollten sie nicht ohne etwas zu lernen gehen.

Als die erste Gruppe gegangen war, führten wir anhand unseres Flowcharts den Reset des Raums durch, trugen im Warteraum die Restzeit von o,oo Minuten ein und da kam auch schon das zweite Team.

Ein Team nach dem anderen ließen wir in den Raum. Wir starrten auf den Laptopmonitor, gaben Tipps über den Bildschirm im Raum, fieberten mit. In manchen Situationen, waren wir in der Schaltzentrale aufgeregter, als die Spieler im Escape Room selbst. Aber wir stellten relativ bald fest, dass die halbe Stunde Zeit für die Anzahl und den Schwierigkeitsgrad der Rätsel wohl zu optimistisch berechnet war. Deshalb ergänzten wir im Highscore bereits nach dem zweiten Team die Spalten „Anzahl gelöster Rätsel“ und „Anzahl Tipps“, um die Vergleichbarkeit der Durchläufe gewährleisten zu können.
Einige wurden im Laufe des Knobelns richtig kreativ. Als Probleme mit den komplexen Zahlen auftauchten, nahm eine Gruppe das Integralbuch von Abramowitz/Stegun in die Hand (was wir nur als falsche Fährte in den Raum gelegt hatten). Doch wir wurden überrascht. Der Student fand nach einigem Blättern das, was er gesucht hatte (die Werte für Sinus und Kosinus an speziellen Stellen) und damit konnte er das Rätsel lösen. Erstaunlich!

Das vorletzte Team für den Tag war dran „Die Algebros ft. Herr C.“. Sie spielten sich souverän durch die Rätsel. Einmal mussten wir den Hinweis geben, dass sie sich nochmal genauer im Raum umschauen sollten, denn sie hatten einen der Schlüssel noch nicht gesehen. Zum Schluss scheiterte es an einem Abschreibefehler, eine falsche Ziffer innerhalb der Matrix. Beinahe hätten sie es geschafft.

Dem letzten Team „Die Freunde des chinesischen Restsatzes" gaben wir noch etwas extra Zeit auf deren Wunsch hin, schließlich mussten wir den Raum nicht mehr zurücksetzen. Auch sie gaben irgendwann auf. Uns blieb nichts anderes übrig als den Highscore mit einer weiteren Restzeit von o,oo Minuten zu vervollständigen.

Auch wenn keines der Teams aus dem Raum entkommen konnte, hatten alle Spaß an der Sache. Auch, dass sie wieder bei einem neuen Escape Room mitmachen würden, freute uns sehr.

\section{Was haben wir jetzt davon?}

Hat sich der Aufwand gelohnt? Um das zu beantworten fassen wir erst einmal den Aufwand der Vorbereitung zusammen: Das Erstellen der Rätsel und das Entwerfen der Flowchartes für den Raum dauerte insgesamt ein paar Stunden, und es mussten ein paar Besorgungen gemacht werden (Schlösser, Kisten, Whiteboardfolie, ...). Die technischen Fragen haben uns etwas mehr Zeit gekostet, aber insgesamt auch nicht mehr als fünf Arbeitsstunden. Probelauf und erster Aufbau des Raums waren noch einmal ein paar Stunden. Insgesamt belief sich der Arbeitsaufwand für die Planung auf weniger als zwei Personentage. Die Durchführung selbst war dann ein ganzer Arbeitstag für zwei Personen und damit konnten ca. 30 Studierende den Raum absolvieren. Unsere Meinung: Das hat sich gelohnt.

Für größere Mengen von Studierenden skaliert sich der Aufwand linear und die Betreuung des Raumes ist einfach, so dass man sehr schnell Hilfskräfte dafür einarbeiten kann (insbesondere können Studierende, die den Raum einmal selbst durchlaufen haben, besonders schnell eingearbeitet
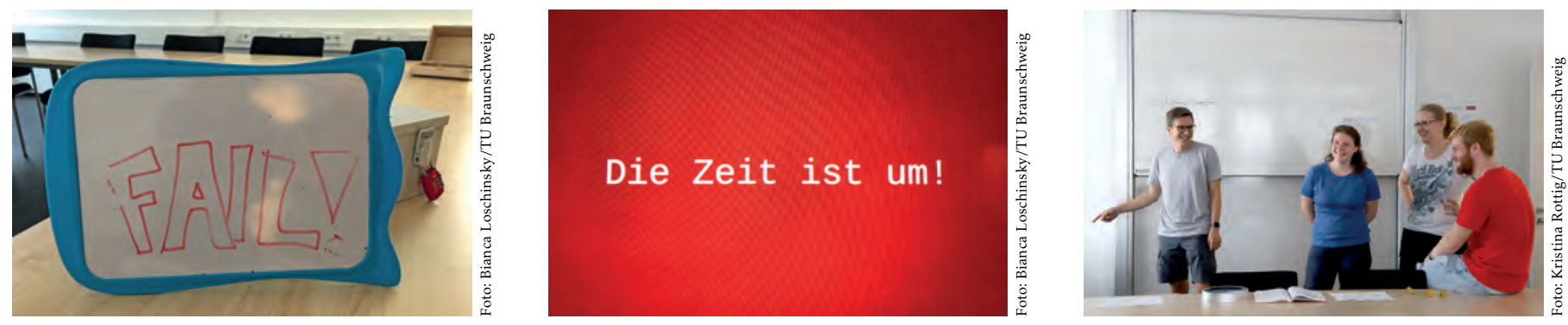


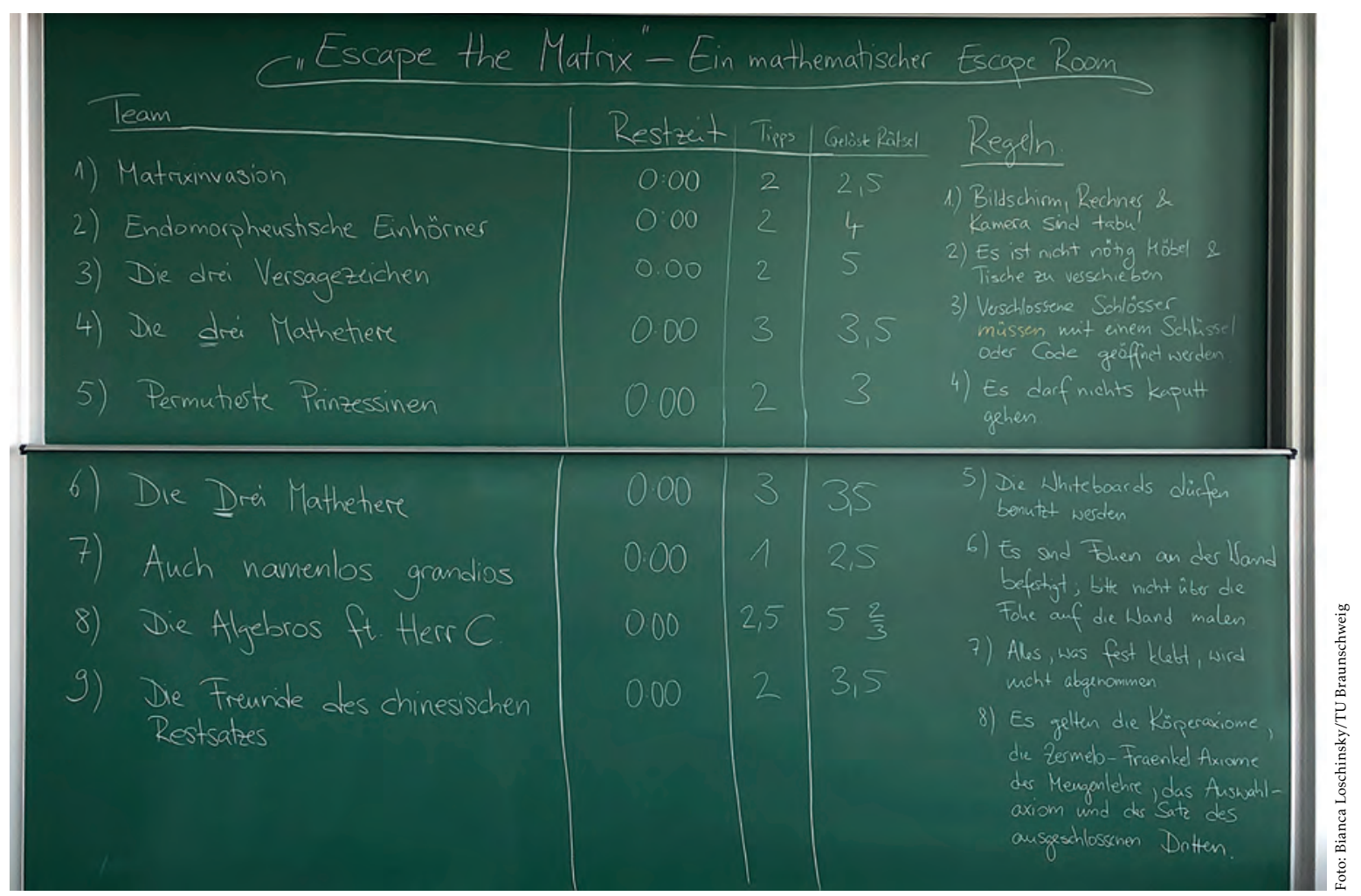

werden und den Raum an einem nächsten Tag selbst betreuen).

Unser Fazit: Ein mathematischer Escape Room ist eine lustige und spannende Ergänzung zum Studium und wir werden in Zukuft auch weitere Escape Rooms für die Anfänger in Mathematik einrichten. Eventuell lohnt es sich auch, solche Räume für große Veranstaltungen in Ingenieursmathematik einzusetzen. Damit könnten solche Veranstaltungen ein frischeres Image bekommen (falls es überhaupt ein Imageproblem gibt ...).

\section{Danksagung}

Wir bedanken uns bei unserem Testteam bestehend aus Fiona Gottschalk, Felix Schneppe und Marko Stautz. Ein weiterer Dank gilt Hinrich Mahler für die Erstellung des Pythonskripts, das für die Hinweisübertragung und den Countdown genutzt wurde. Ein großer Dank gilt allen Teilnehmenden, die es erst ermöglicht haben, dass ein mathematischer Escape Room überhaupt stattfinden konnte.
Anmerkung

1. Natürlich waren die Gruppen aus Sicherheitsgründen nicht wirklich eingeschlossen. Die Tür des Seminarraumes war zwar verschlossen, von Innen aber durch einen Drehknauf jederzeit zu öffnen.

\section{Literatur}

[1] Kerry A. Bartlett and Janice L. Anderson. Gaming to learn: Bringing escape rooms to the classroom. In Handbook of Research on Innovative Digital Practices to Engage Learners, pages 1-27. IGI Global, 2019.

[2] www.hidden-games.de/escape-room-braunschweig/.

[3] Dirk Lorenz. Torus-Origami. Mitt. Dtsch. Math.-Ver., 26(4):204205, 2018.

[4] Alpár István Vita Vörös and Zsuzsa Sárközi. Physics escape room as an educational tool. In AIP Conference Proceedings, volume 1916, page 050002. AIP Publishing, 2017. 